\title{
Author Correction: Tunable two-dimensional interfacial coupling in molecular heterostructures
}

\author{
Beibei Xu1,2, Himanshu Chakraborty ${ }^{3,4}$, Vivek K. Yadav ${ }^{3}$, Zhuolei Zhang ${ }^{1,2}$, Michael L. Klein ${ }^{2,3}$ \& Shenqiang Ren ${ }^{1,2}$
}

Nature Communications 8:312 doi:10.1038/s41467-017-00390-1; Article pubilshed online: 22 Aug 2017

We regretfully omitted to give credit to a previous figure upon which the surface-tension scheme in Fig. $1 \mathrm{~b}$ is based. The caption to Fig. 1 should have included the following: "The surface-tension scheme in Fig. 1b is adapted from Fig. 1a in Noh, J., Jeong, S. \& Lee, J.-Y. Ultrafast formation of air-processable and high-quality polymer films on an aqueous substrate. Nat. Commun. 7, 12374 (2016)."

Published online: 17 November 2017

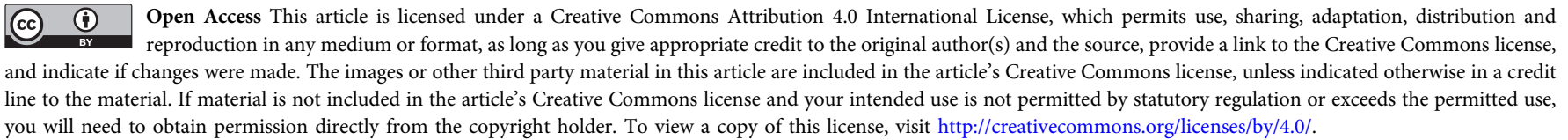

(C) The Author(s) 2017

\footnotetext{
${ }^{1}$ Department of Mechanical Engineering, Temple University, Philadelphia, PA 19122, USA. ${ }^{2}$ Temple Materials Institute, Temple University, Philadelphia, PA 19122, USA. ${ }^{3}$ Department of Chemistry and Institute for Computational Molecular Science, Temple University, Philadelphia, PA 19122, USA. ${ }^{4}$ Center for the Computational Design of Functional Layered Materials, Temple University, Philadelphia, PA 19122, USA. Correspondence and requests for materials should be addressed to S.R. (email: shenqiang.ren@temple.edu)
} 